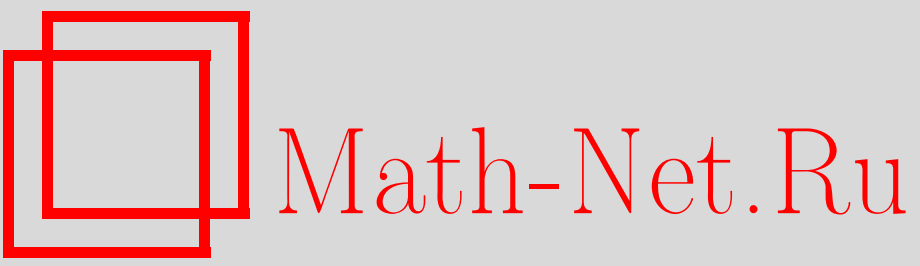

В. В. Сенатов, Об одном асимптотическом разложении в центральной предельной теореме, Теория вероятн. и ее примен., 2011, том 56, выпуск 2, 384-391

DOI: https://doi.org/10.4213/tvp4382

Использование Общероссийского математического портала Math-Net.Ru подразумевает, что вы прочитали и согласны с пользовательским соглашением

http://www.mathnet.ru/rus/agreement

Параметры загрузки:

IP: 54.166 .219 .16

26 апреля 2023 г., 14:39:23 
2. Kramkov D., Schachermayer W. Necessary and sufficient conditions in the problem of optimal investment in incomplete markets. - Ann. Appl. Probab., 2003, v. 13, № 4, p. 1504-1516.

3. Schachermayer $W$. Optimal investment in incomplete markets when wealth may become negative. - Ann. Appl. Probab., 2001, v. 11, № 3, p. 694-734.

4. Schied A., $W u$ C.-T. Duality theory for optimal investments under model uncertainty. - Statist. Decisions, 2005, v. 23, № 3, p. 199-217.

5. Burgert C., Rüschendorf L. Optimal consumption strategies under model uncertainty. - Statist. Decisions, 2005, v. 23, № 1, p. 1-14.

6. Gundel A. Robust utility maximization for complete and incomplete market models. Finance Stoch., 2005, v. 9, № 2, p. 151-176.

7. Föllmer H., Gundel A. Robust projections in the class of martingale measures. Illinois J. Math., 2006, v. 50, № 2, p. 439-472.

8. Гущин $A . A$. Двойственная характеризация цены в задаче максимизации робастной полезности. - Теория вероятн. и ее примен., 2010, т. 55, в. 4, с. 680-704.

9. Морозов И. С. Расширение класса допустимых стратегий в задаче максимизации робастной полезности с конечной на $\mathbf{R}$ функцией полезности. - Обозрение прикл. и промышл. матем., 2010, т. 17, в. 5, с. 617-635.

10. Schied A. Optimal investments for risk- and ambiguity-averse preferences: a duality approach. - Finance Stoch., 2007, v. 11, № 1, p. 107-129.

11. Гущин A. A. О расширении понятия $f$-дивергенции. - Теория вероятн. и ее примен., 2007, т. 52 , в. 3, с. 468-489.

Поступила в редакцию 5.III.2011

(c) 2011 г.

СЕНАТОВ В. В.*

\section{ОБ ОДНОМ АСИМПТОТИЧЕСКОМ РАЗЛОЖЕНИИ В ЦЕНТРАЛЬНОЙ ПРЕДЕЛЬНОЙ ТЕОРЕМЕ ${ }^{1)}$}

Получено асимптотическое разложение, из которого следуют неравномерные оценки как для центральной предельной теоремы, так и для одного из известных асимптотических разложений.

Ключевые слова и фразы: центральная предельная теорема, асимптотические разложения, точность аппроксимации, многочлены Чебышева-Эрмита.

Пусть $X_{1}, X_{2}, \ldots$ - независимые одинаково распределенные случайные величины с нулевым средним, единичной дисперсией и конечным моментом $\beta_{4}=\mathbf{E}\left|X_{1}\right|^{4}$. Предположим, что характеристическая функция $f$ распределения $P$ этих случайных величин такова, что для некоторого числа $\nu>0$

$$
\int_{-\infty}^{\infty}|f(t)|^{\nu} d t<\infty
$$

При выполнении этого условия у распределения нормированной суммы $\left(X_{1}+\cdots+\right.$ $\left.X_{n}\right) / \sqrt{n}$ для $n \geqslant \nu$ существует непрерывная ограниченная плотность $p_{n}$, которую

* Московский государственный университет им. М.В. Ломоносова, механикоматематический факультет, кафедра теории вероятностей, Ленинские горы, 119991 Москва, Россия; e-mail: v.senatov@yandex.ru

1) Работа выполнена при поддержке РФФИ (гранты № 09-01-1217офи_м). 
можно вычислять по формуле обращения

$$
p_{n}(x)=\frac{1}{2 \pi} \int_{-\infty}^{\infty} e^{-i t x} f^{n}\left(\frac{t}{\sqrt{n}}\right) d t, \quad-\infty<x<\infty .
$$

Кроме того, из выполнения условия (1) следует, что $f(t) \rightarrow 0$ при $|t| \rightarrow \infty$ и величина $\alpha(T)=\max \{|f(t)|:|t| \geqslant T\}$ строго меньше единицы для любого $T>0$.

Нам понадобится пара $(\mu, T)$, где функция $\mu(t), e^{-t^{2} / 2} \leqslant \mu(t) \leqslant 1$, и число $T>0$ таковы, что

$$
|f(t)| \leqslant \mu(t) \quad \text { при всех } \quad|t| \leqslant T .
$$

С помощью этой пары определим числа

$$
B_{k, n-j}=\frac{1}{2 \pi} \int_{-T \sqrt{n}}^{T \sqrt{n}}|t|^{k} \mu^{n-j}\left(\frac{t}{\sqrt{n}}\right) d t
$$

$k>0, n \geqslant 3,0 \leqslant j \leqslant n-2$. (Строго говоря, при определении $B_{k, n-j}$ надо было бы использовать третий индекс, скажем $n$ или $j$, но мы этого делать не будем.) Для распределения $P$ с конечным четвертым моментом пару $(\mu, T)$ можно подобрать так, что

$$
B_{k, n-j} \rightarrow B_{k}=\frac{1}{\sqrt{2 \pi}} \int_{-\infty}^{\infty}|x|^{k} \varphi(x) d x
$$

при $n \rightarrow \infty$ для любых фиксированных $k$ и $j$, где $\varphi-$ плотность стандартного нормального закона.

Основная цель этой работы - получить следующее утверждение, которое мы прокомментируем после его доказательства.

Теорема. При $n \geqslant \nu+2$ для всех действительньих $x$

$$
x^{2}\left(p_{n}(x)-\varphi(x)\right)=\frac{\alpha_{3}}{3 ! \sqrt{n}} x^{2} H_{3}(x) \varphi(x)+\frac{1}{2 n}\left(\frac{\alpha_{3}}{3 !}\right)^{2} x^{2} H_{6}(x) \varphi(x)+R,
$$

где $\alpha_{3}=\mathbf{E} X_{1}^{3}-$ третий момент распределения $P$, а

$$
\begin{aligned}
|R| \leqslant & \frac{3}{2 \sqrt{2 \pi}} \frac{\beta_{4}}{n}+\frac{9}{2 \sqrt{2 \pi}}\left(\frac{n}{n-2}\right)^{3 / 2} \frac{1}{n-2}+\frac{\beta_{4}+3}{4 !} \frac{B_{4, n-2}+B_{6, n-3}}{n} \\
& +\frac{3}{\pi} \frac{\left|\alpha_{3}\right|}{n^{3 / 2}}+\frac{\left|\alpha_{3}\right|}{12} \frac{B_{5, n-2}+B_{7, n-3}}{n^{3 / 2}}+\frac{4}{3 \pi} \frac{\left|\alpha_{3}\right| \beta_{4}}{n^{3 / 2}}+\frac{304}{27 \pi} \frac{\left|\alpha_{3}\right|^{3}}{n^{3 / 2}} \\
& +\frac{5}{12 \sqrt{2 \pi}} \frac{\beta_{4}^{2}}{n^{2}}+\frac{1}{2}\left(\frac{\alpha_{3}}{3 !}\right)^{2} \frac{B_{6, n-1}+B_{8, n-2}}{n^{2}} \\
& +\frac{n^{3 / 2}}{\pi}\left(\alpha^{n-\nu-2}(T) \int_{T}^{\infty}|f(t)|^{\nu} d t+\frac{1}{T(n-2)} e^{-T^{2}(n-2) / 2}\right),
\end{aligned}
$$

величина $T>0-$ та же, что в (2).

Здесь и далее мы обозначаем $H_{k}(x)=(-1)^{k} \varphi^{(k)}(x) / \varphi(x), k=0,1, \ldots$, многочлены Чебышёва-Эрмита. Явный вид тех многочленов Чебышёва-Эрмита, которые нам понадобятся, указан к конце доказательства теоремы.

Д о к а з а т е л ь с т в о. Разность $p_{n}(x)-\varphi(x)$ для $n \geqslant \nu$ можно записать в виде

$$
p_{n}(x)-\varphi(x)=\frac{1}{2 \pi} \int_{-\infty}^{\infty} e^{-i t x}\left(f^{n}\left(\frac{t}{\sqrt{n}}\right)-e^{-t^{2} / 2}\right) d t
$$


Умножив это равенство на $(-i x)$ и проинтегрировав по частям, мы видим, что

$$
\begin{aligned}
i x\left(p_{n}(x)-\varphi(x)\right) & =\frac{1}{2 \pi} \int_{-\infty}^{\infty} e^{-i t x}\left(f^{n}\left(\frac{t}{\sqrt{n}}\right)-e^{-t^{2} / 2}\right)^{\prime} d t \\
& =\frac{1}{2 \pi} \int_{-\infty}^{\infty} e^{-i t x}\left(n f^{n-1}\left(\frac{t}{\sqrt{n}}\right) f^{\prime}\left(\frac{t}{\sqrt{n}}\right) \frac{1}{\sqrt{n}}+t e^{-t^{2} / 2}\right) d t .
\end{aligned}
$$

Существование производных функций $f$ вплоть до четвертого порядка гарантируется условием $\beta_{4}<\infty$. Действуя аналогично, мы приходим к равенству

$$
\begin{aligned}
-x^{2}\left(p_{n}(x)-\varphi(x)\right)=\frac{1}{2 \pi} \int_{-\infty}^{\infty} & e^{-i t x}\left((n-1) f^{n-2}\left(\frac{t}{\sqrt{n}}\right)\left(f^{\prime}\left(\frac{t}{\sqrt{n}}\right)\right)^{2}\right. \\
& \left.+f^{n-1}\left(\frac{t}{\sqrt{n}}\right) f^{\prime \prime}\left(\frac{t}{\sqrt{n}}\right)-\left(t^{2}-1\right) e^{-t^{2} / 2}\right) d t
\end{aligned}
$$

При доказательстве этого равенства следует учесть, что $f^{n-1}(t / \sqrt{n}) f^{\prime}(t / \sqrt{n}) \rightarrow 0$ при $|t| \rightarrow \infty$, поскольку $\left|f^{\prime}(t / \sqrt{n})\right| \leqslant \mathbf{E}\left|X_{1}\right| \leqslant 1$ при всех действительных $t$.

Запишем правую часть последнего равенства в виде

$$
\begin{gathered}
\frac{1}{2 \pi} \int_{-\infty}^{\infty} e^{-i t x}\left((n-1) g^{n-2}\left(\frac{t}{\sqrt{n}}\right)\left(f^{\prime}\left(\frac{t}{\sqrt{n}}\right)\right)^{2}-t^{2} e^{-t^{2} / 2}\right) d t \\
+\frac{1}{2 \pi} \int_{-\infty}^{\infty} e^{-i t x}\left(g^{n-1}\left(\frac{t}{\sqrt{n}}\right) f^{\prime \prime}\left(\frac{t}{\sqrt{n}}\right)+e^{-t^{2} / 2}\right) d t+R
\end{gathered}
$$

где $g(t)=e^{-t^{2} / 2} e^{\alpha_{3}(i t)^{3} / 3 !}$ и

$$
\begin{aligned}
|R| \leqslant & \frac{n-1}{2 \pi} \int_{-\infty}^{\infty}\left|f^{n-2}\left(\frac{t}{\sqrt{n}}\right)-g^{n-2}\left(\frac{t}{\sqrt{n}}\right)\right|\left|f^{\prime}\left(\frac{t}{\sqrt{n}}\right)\right|^{2} d t \\
& +\frac{1}{2 \pi} \int_{-\infty}^{\infty}\left|f^{n-1}\left(\frac{t}{\sqrt{n}}\right)-g^{n-1}\left(\frac{t}{\sqrt{n}}\right)\right|\left|f^{\prime \prime}\left(\frac{t}{\sqrt{n}}\right)\right| d t .
\end{aligned}
$$

Так как $\left|f^{\prime}(t / \sqrt{n})\right| \leqslant 1$ и $\left|f^{\prime \prime}(t / \sqrt{n})\right| \leqslant 1$ при всех действительных $t$, то

$$
\begin{aligned}
|R| \leqslant & \frac{n-1}{2 \pi} \int_{-T \sqrt{n}}^{T \sqrt{n}}\left|f^{n-2}\left(\frac{t}{\sqrt{n}}\right)-g^{n-2}\left(\frac{t}{\sqrt{n}}\right)\right|\left|f^{\prime}\left(\frac{t}{\sqrt{n}}\right)\right|^{2} d t \\
& +\frac{1}{2 \pi} \int_{-T \sqrt{n}}^{T \sqrt{n}}\left|f^{n-1}\left(\frac{t}{\sqrt{n}}\right)-g^{n-1}\left(\frac{t}{\sqrt{n}}\right)\right| d t \\
& +\frac{n}{\pi}\left(\int_{T \sqrt{n}}^{\infty}\left|f^{n-2}\left(\frac{t}{\sqrt{n}}\right)\right| d t+\int_{T \sqrt{n}}^{\infty}\left|g^{n-2}\left(\frac{t}{\sqrt{n}}\right)\right| d t\right),
\end{aligned}
$$

где величина $T$ - та же, что в (2). Очевидно, что последнее слагаемое не превосходит

$$
\begin{aligned}
& \frac{n}{\pi}\left(\sqrt{n} \alpha^{n-2-\nu}(T) \int_{T}^{\infty}|f(t)|^{\nu} d t+\int_{T \sqrt{n}}^{\infty} e^{-(n-2) t^{2} /(2 n)} d t\right) \\
& \leqslant \frac{n^{3 / 2}}{\pi}\left(\alpha^{n-2-\nu}(T) \int_{T}^{\infty}|f(t)|^{\nu} d t+\frac{1}{T(n-2)} e^{-T^{2}(n-2) / 2}\right) .
\end{aligned}
$$

Правая часть этого неравенства при росте $n$ убывает экспоненциально быстро. 
Для оценки двух первых слагаемых в правой части (4) воспользуемся хорошо известным равенством

$$
a^{n}-b^{n}=\sum_{j=0}^{n-1} a^{n-j-1} b^{j}(a-b),
$$

справедливым для любых натуральных $n$ и комплексных $a$ и $b$. С помощью этого равенства мы можем второе слагаемое в правой части (4) оценить величиной

$$
\begin{aligned}
& \sum_{j=0}^{n-2} \frac{1}{2 \pi} \int_{-T \sqrt{n}}^{T \sqrt{n}}\left|f^{n-j-2}\left(\frac{t}{\sqrt{n}}\right) g^{j}\left(\frac{t}{\sqrt{n}}\right)\right|\left|f\left(\frac{t}{\sqrt{n}}\right)-g\left(\frac{t}{\sqrt{n}}\right)\right| d t \\
& \leqslant \frac{n-1}{2 \pi} \int_{-T \sqrt{n}}^{T \sqrt{n}} \mu^{n-2}\left(\frac{t}{\sqrt{n}}\right)\left|f\left(\frac{t}{\sqrt{n}}\right)-g\left(\frac{t}{\sqrt{n}}\right)\right| d t .
\end{aligned}
$$

Известно, что при всех действительных $t$

$$
\left|f\left(\frac{t}{\sqrt{n}}\right)-g\left(\frac{t}{\sqrt{n}}\right)\right| \leqslant \frac{\beta_{4}+3}{4 !}\left(\frac{t}{\sqrt{n}}\right)^{4}+\frac{\left|\alpha_{3}\right|}{12}\left|\frac{t}{\sqrt{n}}\right|^{5}+\frac{1}{2}\left(\frac{\alpha_{3}}{3 !}\right)^{2}\left(\frac{t}{\sqrt{n}}\right)^{6} e^{-t^{2} /(2 n)},
$$

это неравенство неоднократно используется в [1, гл. 4]. Поэтому второе слагаемое в правой части (4) не превосходит

$$
\frac{\beta_{4}+3}{4 !} \frac{B_{4, n-2}}{n}+\frac{\left|\alpha_{3}\right|}{12} \frac{B_{5, n-2}}{n^{3 / 2}}+\frac{1}{2}\left(\frac{\alpha_{3}}{3 !}\right)^{2} \frac{B_{6, n-1}}{n^{2}} .
$$

С помощью формулы Тейлора с остаточным членом в интегральной форме легко проверить, что $\left|f^{\prime}(t / \sqrt{n})\right| \leqslant|t / \sqrt{n}|$ при всех действительных $t$. Поэтому первое слагаемое в правой части (4) не превосходит

$$
\begin{aligned}
& \frac{1}{2 \pi} \int_{-T \sqrt{n}}^{T \sqrt{n}} t^{2}\left|f^{n-2}\left(\frac{t}{\sqrt{n}}\right)-g^{n-2}\left(\frac{t}{\sqrt{n}}\right)\right| d t \\
& \quad \leqslant \frac{n-2}{2 \pi} \int_{-T \sqrt{n}}^{T \sqrt{n}} t^{2} \mu^{n-3}\left(\frac{t}{\sqrt{n}}\right)\left|f\left(\frac{t}{\sqrt{n}}\right)-g\left(\frac{t}{\sqrt{n}}\right)\right| d t \\
& \quad \leqslant \frac{\beta_{4}+3}{4 !} \frac{B_{6, n-3}}{n}+\frac{\left|\alpha_{3}\right|}{12} \frac{B_{7, n-3}}{n^{3 / 2}}+\frac{1}{2}\left(\frac{\alpha_{3}}{3 !}\right)^{2} \frac{B_{8, n-2}}{n^{2}} .
\end{aligned}
$$

Очевидно,что первое слагаемое в (3) можно записать в виде

$$
\frac{1}{2 \pi} \int_{-\infty}^{\infty} e^{-i t x}\left(n g^{n}\left(\frac{t}{\sqrt{n}}\right)\left(f^{\prime}\left(\frac{t}{\sqrt{n}}\right)\right)^{2}-t^{2} e^{-t^{2} / 2}\right) d t+R
$$

где

$$
\begin{aligned}
|R| & \leqslant \frac{1}{2 \pi} \int_{-\infty}^{\infty}\left|g^{n-2}\left(\frac{t}{\sqrt{n}}\right)\right|\left|f^{\prime}\left(\frac{t}{\sqrt{n}}\right)\right|^{2}\left|(n-1)-n g^{2}\left(\frac{t}{\sqrt{n}}\right)\right| d t \\
& \leqslant \frac{1}{2 \pi} \int_{-\infty}^{\infty} t^{2} e^{-(n-2) t^{2} /(2 n)}\left(\left|g^{2}\left(\frac{t}{\sqrt{n}}\right)-1\right|+\frac{1}{n}\right) d t .
\end{aligned}
$$

Ясно, что

$$
\begin{aligned}
g^{2}\left(\frac{t}{\sqrt{n}}\right)-1 & =e^{-t^{2} / n} \exp \left\{\frac{\alpha_{3}}{3}\left(\frac{i t}{\sqrt{n}}\right)^{3}\right\}-1=e^{-t^{2} / n}\left(1+\gamma \frac{\alpha_{3}}{3}\left(\frac{t}{\sqrt{n}}\right)^{3}\right)-1 \\
& =e^{-t^{2} / n}-1+\gamma \frac{\alpha_{3}}{3}\left(\frac{t}{\sqrt{n}}\right)^{3} e^{-t^{2} / n}
\end{aligned}
$$


Здесь и далее $\gamma$ обозначает непрерывные комплекснозначные функции такие, что $|\gamma| \leqslant$

1. Эти функции в разных местах, вообще говоря, различны.

Теперь нетрудно проверить, что модуль последней величины $R$ не превосходит

$$
\begin{aligned}
& \frac{1}{2 \pi} \int_{-\infty}^{\infty} t^{2} e^{-(n-2) t^{2} /(2 n)}\left(\frac{1}{n}+\frac{t^{2}}{n}+e^{-t^{2} / n} \frac{\left|\alpha_{3}\right|}{3}\left|\frac{t}{\sqrt{n}}\right|^{3}\right) d t \\
& \leqslant \frac{1}{\sqrt{2 \pi}}\left(\frac{n}{n-2}\right)^{3 / 2}\left(\frac{1}{n}+\frac{3}{n-2}\right)+\frac{\left|\alpha_{3}\right|}{3} \frac{B_{5}}{n^{3 / 2}} \\
& \leqslant \frac{4}{\sqrt{2 \pi}}\left(\frac{n}{n-2}\right)^{3 / 2} \frac{1}{n-2}+\frac{\left|\alpha_{3}\right|}{3} \frac{B_{5}}{n^{3 / 2}} .
\end{aligned}
$$

Второе слагаемое в (3) можно записать в виде

$$
\frac{1}{2 \pi} \int_{-\infty}^{\infty} e^{-i t x}\left(g^{n}\left(\frac{t}{\sqrt{n}}\right) f^{\prime \prime}\left(\frac{t}{\sqrt{n}}\right)+e^{-t^{2} / 2}\right) d t+R
$$

где

$$
\begin{aligned}
|R| & \leqslant \frac{1}{2 \pi} \int_{-\infty}^{\infty} e^{-(n-1) t^{2} /(2 n)}\left|f^{\prime \prime}\left(\frac{t}{\sqrt{n}}\right)\right|\left|1-g\left(\frac{t}{\sqrt{n}}\right)\right| d t \\
& \leqslant \frac{1}{2 \pi} \int_{-\infty}^{\infty} e^{-(n-1) t^{2} /(2 n)}\left(\frac{t^{2}}{2 n}+e^{-t^{2} /(2 n)}\left|\frac{\alpha_{3}}{3 !}\right|\left|\frac{t}{\sqrt{n}}\right|^{3}\right) d t \\
& =\frac{1}{2 \sqrt{2 \pi} n}\left(\frac{n}{n-1}\right)^{3 / 2}+\frac{\left|\alpha_{3}\right|}{3 !} \frac{B_{3}}{n^{3 / 2}} .
\end{aligned}
$$

По формуле Тейлора с остаточным членом в интегральной форме,

$$
\begin{aligned}
f^{\prime}\left(\frac{t}{\sqrt{n}}\right) & =f^{\prime}(0)+f^{\prime \prime}(0) \frac{t}{\sqrt{n}}+\frac{1}{2} f^{\prime \prime \prime}(0)\left(\frac{t}{\sqrt{n}}\right)^{2}+\frac{\gamma}{3 !} \beta_{4}\left(\frac{t}{\sqrt{n}}\right)^{3} \\
& =-\frac{t}{\sqrt{n}}+\frac{i \alpha_{3}}{2}\left(\frac{i t}{\sqrt{n}}\right)^{2}+\frac{\gamma}{3 !} \beta_{4}\left(\frac{t}{\sqrt{n}}\right)^{3}
\end{aligned}
$$

$$
f^{\prime \prime}\left(\frac{t}{\sqrt{n}}\right)=f^{\prime \prime}(0)+f^{\prime \prime \prime}(0) \frac{t}{\sqrt{n}}+\frac{\gamma}{2} \beta_{4}\left(\frac{t}{\sqrt{n}}\right)^{2}=-1-\alpha_{3}\left(\frac{i t}{\sqrt{n}}\right)+\frac{\gamma}{2} \beta_{4}\left(\frac{t}{\sqrt{n}}\right)^{2} .
$$

Поэтому первое слагаемое в (5) равно

$$
\begin{gathered}
\frac{1}{2 \pi} \int_{-\infty}^{\infty} e^{-i t x}\left(n e ^ { - t ^ { 2 } / 2 } \operatorname { e x p } \{ \frac { \alpha _ { 3 } } { 3 ! } \frac { ( i t ) ^ { 3 } } { \sqrt { n } } \} \left(\left(\frac{t}{\sqrt{n}}\right)^{2}-\left(\frac{\alpha_{3}}{2}\right)^{2}\left(\frac{i t}{\sqrt{n}}\right)^{4}\right.\right. \\
\left.\left.-\alpha_{3}\left(\frac{i t}{\sqrt{n}}\right)^{3}\right)-t^{2} e^{-t^{2} / 2}\right) d t+R,
\end{gathered}
$$

где

$$
\begin{aligned}
|R| & \leqslant \frac{1}{2 \pi} \int_{-\infty}^{\infty} n e^{-t^{2} / 2}\left(\left(\frac{\beta_{4}}{3 !}\right)^{2}\left(\frac{t}{\sqrt{n}}\right)^{6}+\frac{\beta_{4}}{3}\left(\frac{t}{\sqrt{n}}\right)^{4}+\frac{\left|\alpha_{3}\right| \beta_{4}}{3 !}\left|\frac{t}{\sqrt{n}}\right|^{5}\right) d t \\
& \leqslant\left(\frac{\beta_{4}}{3 !}\right)^{2} \frac{B_{6}}{n^{2}}+\frac{\beta_{4}}{3} \frac{B_{4}}{n}+\frac{\left|\alpha_{3}\right| \beta_{4}}{3 !} \frac{B_{5}}{n^{3 / 2}} .
\end{aligned}
$$


Первое слагаемое в (7) равно сумме трех величин

$$
\begin{aligned}
& \frac{1}{2 \pi} \int_{-\infty}^{\infty} e^{-i t x} e^{-t^{2} / 2}\left(n \exp \left\{\frac{\alpha_{3}}{3 !} \frac{(i t)^{3}}{\sqrt{n}}\right\}\left(\frac{t}{\sqrt{n}}\right)-t^{2}\right)^{2} d t, \\
& -\frac{1}{2 \pi} \int_{-\infty}^{\infty} e^{-i t x} e^{-t^{2} / 2} n \exp \left\{\frac{\alpha_{3}}{3 !} \frac{(i t)^{3}}{\sqrt{n}}\right\}\left(\frac{\alpha_{3}}{2}\right)^{2}\left(\frac{i t}{\sqrt{n}}\right)^{4} d t, \\
& -\frac{1}{2 \pi} \int_{-\infty}^{\infty} e^{-i t x} e^{-t^{2} / 2} n \exp \left\{\frac{\alpha_{3}}{3 !} \frac{(i t)^{3}}{\sqrt{n}}\right\} \alpha_{3}\left(\frac{i t}{\sqrt{n}}\right)^{3} d t .
\end{aligned}
$$

Эти величины суть

$$
\begin{aligned}
& \frac{1}{2 \pi} \int_{-\infty}^{\infty} e^{-i t x} e^{-t^{2} / 2} t^{2}\left(\frac{\alpha_{3}}{3 !} \frac{(i t)^{3}}{\sqrt{n}}+\frac{1}{2}\left(\frac{\alpha_{3}}{3 !}\right)^{2} \frac{(i t)^{6}}{n}+\frac{\gamma}{3 !}\left(\frac{\alpha_{3}}{3 !}\right)^{3} \frac{t^{9}}{n^{3 / 2}}\right) d t \\
- & \frac{1}{2 \pi} \int_{-\infty}^{\infty} e^{-i t x} e^{-t^{2} / 2}\left(1+\gamma \frac{\alpha_{3}}{3 !} \frac{t^{3}}{\sqrt{n}}\right)\left(\frac{\alpha_{3}}{2}\right)^{2} \frac{(i t)^{4}}{n} d t \\
- & \frac{1}{2 \pi} \int_{-\infty}^{\infty} e^{-i t x} e^{-t^{2} / 2}\left(1+\frac{\alpha_{3}}{3 !} \frac{(i t)^{3}}{\sqrt{n}}+\frac{\gamma}{2}\left(\frac{\alpha_{3}}{3 !}\right)^{2} \frac{t^{6}}{n}\right) \alpha_{3} \frac{(i t)^{3}}{\sqrt{n}} d t
\end{aligned}
$$

Так как для любого натурального $k$ справедлива формула обращения

$$
\frac{1}{2 \pi} \int_{-\infty}^{\infty} e^{-i t x} e^{-t^{2} / 2}(i t)^{k} d t=H_{k}(x) \varphi(x),
$$

то первое слагаемое в (7) равно

$$
\begin{aligned}
& -\frac{\alpha_{3}}{3 ! \sqrt{n}} H_{5}(x) \varphi(x)-\frac{1}{2 n}\left(\frac{\alpha_{3}}{3 !}\right)^{2} H_{8}(x) \varphi(x) \\
& -\left(\frac{\alpha_{3}}{2}\right)^{2} \frac{1}{n} H_{4}(x) \varphi(x)-\frac{\alpha_{3}}{\sqrt{n}} H_{3}(x) \varphi(x)-\alpha_{3} \frac{\alpha_{3}}{3 ! n} H_{6}(x) \varphi(x)+R,
\end{aligned}
$$

где

$$
|R| \leqslant \frac{1}{3 !}\left|\frac{\alpha_{3}}{3 !}\right|^{3} \frac{B_{11}}{n^{3 / 2}}+\frac{1}{3}\left|\frac{\alpha_{3}}{2}\right|^{3} \frac{B_{7}}{n^{3 / 2}}+\frac{\left|\alpha_{3}\right|}{2}\left(\frac{\alpha_{3}}{3 !}\right)^{2} \frac{B_{9}}{n^{3 / 2}} .
$$

Первое слагаемое в (6) равно

$$
\frac{1}{2 \pi} \int_{-\infty}^{\infty} e^{-i t x} e^{-t^{2} / 2}\left(\exp \left\{\frac{\alpha_{3}}{3 !} \frac{(i t)^{3}}{\sqrt{n}}\right\}\left(-1-\alpha_{3} \frac{i t}{\sqrt{n}}\right)+1\right) d t+R
$$

где

$$
|R| \leqslant \frac{1}{2 \pi} \int_{-\infty}^{\infty} e^{-t^{2} / 2} \frac{\beta_{4}}{2}\left(\frac{t}{\sqrt{n}}\right)^{2} d t=\frac{\beta_{4}}{2 \sqrt{2 \pi} n} .
$$

Первое слагаемое в (11) равно

$$
\begin{aligned}
-\frac{1}{2 \pi} \int_{-\infty}^{\infty} e^{-i t x} e^{-t^{2} / 2}( & \frac{\alpha_{3}}{3 !} \frac{(i t)^{3}}{\sqrt{n}}+\frac{1}{2}\left(\frac{\alpha_{3}}{3 !}\right)^{2} \frac{(i t)^{6}}{n}+\frac{\gamma}{3 !}\left(\frac{\alpha_{3}}{3 !}\right)^{3} \frac{t^{9}}{n^{3 / 2}} \\
& \left.+\alpha_{3}\left(\frac{i t}{\sqrt{n}}\right)+\alpha_{3} \frac{\alpha_{3}}{3 !} \frac{(i t)^{4}}{n}+\frac{\gamma}{2} \alpha_{3}\left(\frac{\alpha_{3}}{3 !}\right)^{2} \frac{t^{7}}{n^{3 / 2}}\right) d t \\
=-\frac{\alpha_{3}}{3 ! \sqrt{n}} H_{3}(x) \varphi(x) & -\frac{1}{2 n}\left(\frac{\alpha_{3}}{3 !}\right)^{2} H_{6}(x) \varphi(x)-\frac{\alpha_{3}}{\sqrt{n}} H_{1}(x) \varphi(x)-\frac{\alpha_{3}^{2}}{3 ! n} H_{4}(x) \varphi(x)+R
\end{aligned}
$$


где

$$
|R| \leqslant \frac{1}{3 !}\left|\frac{\alpha_{3}}{3 !}\right|^{3} \frac{B_{9}}{n^{3 / 2}}+\frac{\left|\alpha_{3}\right|}{2}\left(\frac{\alpha_{3}}{3 !}\right)^{2} \frac{B_{7}}{n^{3 / 2}} .
$$

Собирая вместе величины, содержащие многочлены Чебышёва-Эрмита, мы видим, что их сумма равна

$$
\begin{aligned}
- & \frac{\alpha_{3}}{3 ! \sqrt{n}}\left(H_{5}(x)+6 H_{3}(x)+H_{3}(x)+6 H_{1}(x)\right) \varphi(x) \\
& -\frac{1}{2 n}\left(\frac{\alpha_{3}}{3 !}\right)^{2}\left(H_{8}(x)+18 H_{4}(x)+12 H_{6}(x)+H_{6}(x)+12 H_{4}(x)\right) \varphi(x) .
\end{aligned}
$$

Используя равенства

$$
\begin{gathered}
H_{1}(x)=x, \quad H_{3}(x)=x^{3}-3 x, \quad H_{4}(x)=x^{4}-6 x^{2}+3, \quad H_{5}(x)=x^{5}-10 x^{3}+15 x, \\
H_{6}(x)=x^{6}-15 x^{4}+45 x^{2}-15, \quad H_{8}(x)=x^{8}-28 x^{6}+210 x^{4}-420 x^{2}+105,
\end{gathered}
$$

нетрудно проверить, что сумма (12) с точностью до знака совпадает с главной частью

$$
\frac{\alpha_{3}}{3 ! \sqrt{n}} x^{2} H_{3}(x) \varphi(x)+\frac{1}{2 n}\left(\frac{\alpha_{3}}{3 !}\right)^{2} x^{2} H_{6}(x) \varphi(x)
$$

разложения величины $x^{2}\left(p_{n}(x)-\varphi(x)\right)$, указанной в формулировке теоремы. Собирая вместе неравенства, полученные по ходу построения асимптотического разложения, и используя равенства $B_{k+2}=(k+1) B_{k}, k=0,1, \ldots, B_{3}=2 / \pi$ и $B_{2}=1 / \sqrt{2 \pi}$, нетрудно убедиться в справедливости оценки теоремы для остаточной части разложения.

В работе [1] при тех же условия, что использовались в доказанной теореме, было получено следующее асимптотическое разложение с равномерной оценкой остаточной части (теорема $1 \S 9$ из главы 4):

$$
p_{n}(x)=\varphi(x)+\frac{\alpha_{3}}{3 ! \sqrt{n}} H_{3}(x) \varphi(x)+\frac{1}{2 n}\left(\frac{\alpha_{3}}{3 !}\right)^{2} H_{6}(x) \varphi(x)+R,
$$

где

$$
\begin{aligned}
|R| \leqslant & \frac{\beta_{4}+3}{4 !} \frac{B_{4, n-1}}{n}+\frac{\left|\alpha_{3}\right|}{12} \frac{B_{5, n-1}}{n^{3 / 2}}+\frac{1}{3 !}\left|\frac{\alpha_{3}}{3 !}\right|^{3} \frac{B_{9}}{n^{3 / 2}} \\
& +\frac{1}{2}\left(\frac{\alpha_{3}}{3 !}\right)^{2} \frac{B_{6, n}}{n^{2}}+\frac{\sqrt{n}}{\pi} \alpha^{n-\nu}(T) \int_{T}^{\infty}|f(t)|^{\nu} d t+\frac{1}{\pi T \sqrt{n}} e^{-T^{2} n / 2} .
\end{aligned}
$$

Это разложение относится к так называемым уточненным разложениям ЭджвортаКрамера. Равенство (13) в какой-то мере позволяет судить о естественности разложения функции $x^{2}\left(p_{n}(x)-\varphi(x)\right)$, полученного выше. Ясно, что утверждение доказанной нами теоремы дает для $x \neq 0$ неравномерную оценку разности

$$
p_{n}(x)-\left\{\varphi(x)+\frac{\alpha_{3}}{3 ! \sqrt{n}} H_{3}(x) \varphi(x)+\frac{1}{2 n}\left(\frac{\alpha_{3}}{3 !}\right)^{2} H_{6}(x) \varphi(x)\right\}
$$

- ее абсолютная величина не превосходит оценки модуля величины $R$, приведенной в формулировке теоремы, деленной на $x^{2}$. Степень 2 при $x$ не является оптимальной, при конечности величины $\beta_{4}$ справедлив аналог полученного разложения, в котором множитель $x^{2}$ при $p_{n}(x)-\varphi(x)$ заменяется на $x^{4}$, но оценка остаточной части разложения становится значительно более громоздкой. 


\section{СПИСОК ЛИТЕРАТУРЫ}

1. Сенатов B. В. Центральная предельная теорема: точность аппроксимации и асимптотические разложения. М.: Книжный дом «Либроком», 2009, 352 с.

Поступила в редакцию 12.IX.2008

(c) 2011 г.

СИНЕЛЬНИКОВ С. С.*

\section{ОБ ОПТИМАЛЬНОЙ ОСТАНОВКЕ БРОУНОВСКОГО ДВИЖЕНИЯ С ОТРИЦАТЕЛЬНЫМ СНОСОМ}

Для броуновского движения с отрицательным сносом на интервале $[0, \infty)$ рассматриваются задачи об оптимальной остановке, связанные с «наилучшим» оцениванием двух непредсказуемых моментов: момента, при котором достигается максимальное значение, и момента достижения последнего нуля процесса.

Ключевые слова и фразы: непредсказуемый момент, скорейшее обнаружение, броуновское движение со сносом.

1. Постановка задачи. Пусть $B_{t}^{\mu}$, где $\mu<0$, - стандартное броуновское движение с отрицательным сносом на бесконечном интервале, $t \in[0, \infty)$. Рассматриваются следующие непредсказуемые моменты:

$$
\theta=\inf \left\{t: B_{t}^{\mu}=\sup _{s \geqslant 0} B_{s}^{\mu}\right\}, \quad g=\sup \left\{t: B_{t}^{\mu}=0\right\} .
$$

Таким образом, $\theta$ - это момент достижения абсолютного максимума процессом $B_{t}^{\mu}$, a $g$ - последний момент достижения процессом значения 0. Поскольку снос $\mu$ отрицателен, то моменты $\theta$ и $g$ являются конечными почти наверное. Нас интересует оптимальное приближение этих моментов на основе информации, доступной на текущий момент. В качестве критериев оптимальности приближения в данной работе рассматриваются два критерия: условно-экстремальный и абсолютный.

Зафиксируем $\alpha \in(0,1)$ и определим следующие классы моментов остановки:

$$
\mathscr{M}_{\alpha}^{B^{\mu}}(\theta)=\left\{\tau \in \mathscr{M}^{B^{\mu}}: \mathbf{P}(\tau<\theta) \leqslant \alpha\right\}, \quad \mathscr{M}_{\alpha}^{B^{\mu}}(g)=\left\{\tau \in \mathscr{M}^{B^{\mu}}: \mathbf{P}(\tau<g) \leqslant \alpha\right\},
$$

где $\mathscr{M}^{B^{\mu}}$ - класс марковских моментов, порожденных рассматриваемым процессом. Под моментами, являющимися решением задачи при условно-экстремальном критерии, мы понимаем такие моменты $\theta^{*} \in \mathscr{M}_{\alpha}^{B^{\mu}}(\theta)$ и $g^{*} \in \mathscr{M}_{\alpha}^{B^{\mu}}(g)$, что

$$
\begin{aligned}
& \mathbf{E}\left(\theta^{*}-\theta\right)^{+}=\inf _{\tau \in \mathscr{M}_{\alpha}^{B}{ }^{\mu}(\theta)} \mathbf{E}(\tau-\theta)^{+}, \\
& \mathbf{E}\left(g^{*}-g\right)^{+}=\inf _{\tau \in \mathscr{M}_{\alpha}^{B^{\mu}}(g)} \mathbf{E}(\tau-g)^{+},
\end{aligned}
$$

где $a^{+}=a$, если $a \geqslant 0$, и $a^{+}=0$, если $a<0$.

Под моментами, являющимися решением задачи при абсолютном критерии, мы понимаем такие моменты $\theta^{*} \in \mathscr{M}^{B^{\mu}}$ и $g^{*} \in \mathscr{M}^{B^{\mu}}$, что

$$
\mathbf{E}\left|\theta^{*}-\theta\right|=\inf _{\tau \in \mathscr{M}^{B}{ }^{\mu}} \mathbf{E}|\tau-\theta|, \quad \mathbf{E}\left|g^{*}-g\right|=\inf _{\tau \in \mathscr{M}^{B}{ }^{\mu}} \mathbf{E}|\tau-g| .
$$

* Московский государственный университет им. М.В. Ломоносова, механикоматематический факультет, кафедра теории вероятностей, Ленинские горы, 119991 Москва, Россия; е-mail: sergesinelnikov@gmail.com 